dorsum. The tracing (Fig. 2), made from a radiogram of a dissection, in which the dorsal cutaneous branch has been isolated in the neighbourhood of the wrist and painted with a material which will give a shadow, shows the normal intimate relation of the nerve to the bone. From a number of dissections it appears that the branch occasionally may curve on to the dorsum distal to the lower end of the ulna, in which position consequently the former is less exposen to compression by any constriction at the wrist.

Dangers of Constriction.-These cases emphasise the clinical significance of neuro-osseous relations, and the necessity to avoid any form of constriction or pressure at points where nerves lie upon bone. Several times $I$ have seen, in the distribution of the musculo-

Frg. 1.

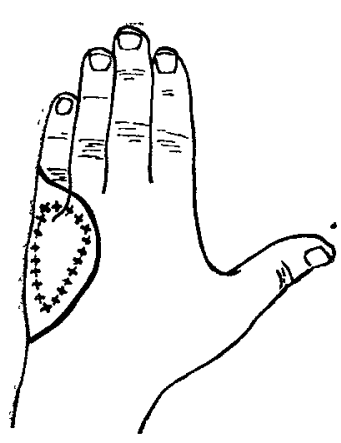

To show the loss of sensation in the tinuous line $=$ anæs. thesia to lightest touch. Crosses $=$ analgesiato pin prick.

utaneous nerve of the leg, clinical evidence of changes in the nerve, apparently due to compression by a tight sock-suspender or other form of constriction below the knee. In every case the manifestations of neuritis have been discovered more or less accidentally in the course of a general examination of the nervous system, and none complained of subjective symptoms in this region; on inquiry a small proportion stated that they had occasionally suffered from tingling or a similar sensation in the outer part of the leg and on the dorsum of the foot, but it was obvious that none had experienced very much pain. The objective manifestations were both sensory and trophic. The former ranged from small patches of hypoæsthesia to areas of ancesthesia and analgesia corresponding almost to the full cutaneous distribution of the musculocutaneous nerve.

Trophic Changes.-Trophic changes were more contantly discovered and consisted of loss of hair, loss of cutaneous elasticity, and greater smoothness of the skin in the distribution of the affected nerve. In a few the absence of hair accurately marked out the cutaneous distribution of the musculo-cutaneous, and the difficulty of raising the skin from the deeper tissues of this region, compared to the ease with which it was elevated on the inner aspect of the leg, was very striking. No patient exhibited any demonstrable paresis in any of the anterior tibial or peroneal groups of muscles. Invariably the objective sensory and trophic manifestations were confined to the distribution of the musculo-cutaneous, and did not extend to the regions supplied by the sural branch of the external popliteal or the external saphenous, which is a point of diagnostic value in localising the anatomical level of the cause of the symptoms. In every patient signs were discovered in both legs, but commonly they were more pronounced on one side.

Although the symptoms were always confined to the distribution of the musculo-cutaneous, there seems little doubt that the compression must be exerted upon the external popliteal, where it curves round the outer side of the neck of the fibula. At this point the external popliteal is very exposed, being in contact with bone and covered only by skin and aponeurosis, whereas the musculo-cutaneous is much more protected since it lies in the peroneus longus.

In spite of the fact that no very serious disturbances have been seen as a result of the compression, it seems possible that trophic ulcers might easily develop and lead to serious consequences, and for this reason it is necessary to realise that compression of the external popliteal by a constriction below the knee may be a predisposing cause of ulceration in the outer part of the leg or on the dorsum of the foot.

\section{Climital Altotes:}

MEDICAL, SURGICAL, OBSTETRICAL, AND THERAPEUTICAL.

\section{A CASE OF}

GIANT URTICARIA TREATED BY AN AUTO. GENOUS STREPTOCOCCUS VACCINE.

By W. E. M. Armstrong, M.A., M.D., BACTERIOLOGIST, CENTRAL LONDON OPHTHALMIC HOSPITAL.

THIs case appeared to be genuine giant urticaria, and occurred in a female patient aged 31 . She said the disease began ten years before after having eaten a dish of mushrooms.

Signs and Symptoms.- Eivery four or five days a red rash appeared, rapidly reached a maximum degree of intensity and extent, and usually subsided almost completely within 48 hours of the first onset. The rash was accompanied by a temperature which often rose as high as $102.5^{\circ} \mathbf{F}$., and by a considerable amount of headache and general malaise. The eruption presented several different characters, which were apparently only different degrees of one and the same pathological process. It followed no definite plan of distribution, but developed on any part of the face, body, or limbs, showing no predilection for any particular anatomical locality. The different features shown graded gradually into one another, suggesting that the differences were of degree and not of kind.

First small patches of erythema appeared : these were usually multiple, varying in size from spots like erythema nodosum to large, flat, map-like areas with irregular motheaten edges not well differentiated from the surrounding skin. These patches sometimes subsided, but more often became larger and more boldly outlined, at the same time taking on an urticarial character. This urticaria frequently reached a very extreme degree and caused large increases in the size of the affected parts, which had the appearance of a cellulitis, though the skin remained soft to the touch and showed little or no signs of the brawniness incidental to an erysipelatous condition. When the rash faded it left behind a scaly peeling surface. These phenomena could be partially induced by artificial means. A light blow or the exertion of pressure on any part of the patient's body produced an urticarial swelling of greater or less degree according to the amount of violence exerted.

History.-The patient's history, and that of her family, did not afford much clue to the nature of the disorder, but there was a definite history of rheumatism in the family, and she herself suffered from this complaint and from sore-throats.

As the patient had had all the ordinary treatments with no benefit, it only remained to see if some method of immunisation could be devised. The selection of a likely antigen was accordingly the first practical consideration. The recurrent temperature pointed to a bacterial origin The combination of sore-throats with rheumatism, the likeness of the patches to erysipelas, and the peeling of the epidermis which nearly always followed the eruption, suggested that an organism of streptococcal type might be the cause of the trouble.

Clinical Examination.-No obvious source of infection could be discovered. The Wassermann reaction was negative and in other respects the blood appeared normal. A blood culture was not undertaken because it was improbable, from the low range of temperature and the comparatively slight constitutional disturbances, that the bacterial invasion of the blood stream attained a sufficiently high concentration to render positive findings likely by this method of search. A catheter specimen of urine was obtained when the patient was in the middle of an attack. From this a small badlystaining short streptococcus was isolated. This was grown with some difficulty aerobically on trypsin-agar and a vaccine prepared. 
Treatmenl. - This was begun by the inoculation of a pilot dose of $1,000,000$ dead streptococci. This measure was promptiy followed by an attack of rheumatism which lasted for six days. There was no exacerbation of the rash; on the contrary, the latter was very much improved, and continued so for seven days after the inoculation. A similar dose was then given, which did not produce rheumatism, but did produce an immediate and very violent outbreak of the skin condition which lasted for 13 days. This negative phase was followed by a positive phase lasting 26 days, during which the skin remained almost well. The doses were then reduced to 100,000 streptococci. Only two of these were required, the second being administered in December, 1920. Since then there has been no recurrence of either the rheumatism or the skin trouble, and the cure appears to be an absolute one.

The case is worthy of note because of the uncommon symptoms, and also because it demonstrates how a disease which had resisted treatment by various methods for a period of ten years yielded almost at once to immunisation methods appropriately employed. It is also interesting to see that the same bacterium could apparently produce, in the same patient, such clinically dissimilar conditions as rheumatism and urticaria.

\section{COLLOIDAL ANTIMONY IN LEISHMANIA INFANTUM.}

By Robert Samut, M.B., C.M. Edin., PROFESSOR OF PATHOLOGY, UNIVERSTTY OF MALTA.

EncourageD by the successful results obtained in Hodgkin's disease by the use of colloidal manganese, I employed colloidal antimony in a case of leishmania infantum; although the patient's death from purely accidental circumstances prevented me from observing the ultimate result of the treatment, I am convinced that the intramuscular injection of colloidal antimony is of such great advantage over the intravenous introduction of the ordinary solution of tartar emetic that I would recommend its further use in all cases of this disease.

C. B. was first brought to me suffering from profound anxmia. I diagnosed the case as one of leukremia, since the spleen was enlarged, but omitted the microscopical examination of the blood. I treated the patient with liq. arsenicalis, syr. ferri phosph. co., and glycer. medullæ ossis. The child improved and $I$ then lost sight of her until the beginning of 1921. She was now 4 years of age ; a pale, puny, emaciated child with a huge abdomen, an enormously enlarged spleen, a slightly enlarged liver, and petechiæ all over her body. The extreme dark-lemon colour of the skin stood out as a well-pronounced feature in the case. The temperature often ran up to $105^{\circ}$ F. and dropped again to normal in a few hours, while the child was seemingly unaffected by the high temperature. The feet swelled sometimes, but not to any marked degree. Leishman-Donovan bodies were present in the blood obtained by puncture from the liver, but were absent from the lymph of a small blister raised for the purpose. The majority of these bodies were enclosed in polymorphonuclear leucocytes; some were free in the plasma. The mononuclear leucocytes (Iymphocytes) were increased in number, the following being the blood picture :-

$\mathrm{Hr}$

Hb $\ldots \quad \ldots \quad \ldots 0 \%$ Large lymphocytes

Wrythrocytes 2,850,000 per c.mm. Fosinophiles

Colour index

12,000

Polymorphonuclears

mall lymphocytes

Basophiles

$25 \%$

‥ $12 \%$ Myelocytes $\cdots \cdots$ Nil. injections of $1 \mathrm{c.cm}$. of a 1 per cent. solution of tartar emetic. I chose the occipito-temporal veins as the seat for injection, but was obliged to give up the attempt at once as the patient refused to submit to the treatment. I then determined to use the colloidal form of antimony and began treatment, using phials prepared by Messrs. Chatelain, of the Laboratoire des Colloildes, 2, Rue de Valenciennes, the sulphide of antimony in $1 \mathrm{~cm}$ of distilled water phial containing $3 \mathrm{ccm}$. of the solution. I injected the contents of one phial deeply into the muscles of the buttock, using one phial daily on alternate weeks.

In a fortnight the spleen had decreased in size, and in about four weeks it had retreated to the left of the mesial plane of the body; prior to the treatment it was fully 2 inches to the right of this plane. Further injections produced further improvement. But one night the patient had a terrible attack of epistaxis; surgical help could not be obtained for several hours, and the child lost enormous quantities of blood. When I saw her she was almost bloodless and suffering from dyspnoea and slight delirium. She sank gradually and died about five days after the hæmorrhage.

Her general health had greatly improred. The size of the abdomen had decreased visibly with the fall in size of the spleen; her colour had also improved. She relished her food, was fond of play, and was able to endure the fatigue of " a walk," while prior to the treatment she was practically unable to get about. It will thus be seen that the improvement in the child's condition could be due to no other factor except the treatment employed.

A CASE OF

A C UT E A S A R I A I S

ASSOCIATED WITI TRICHURIS TRICHIURA COMPLICATING PREGNANCY.

\section{By C. Russell Corfield, M.D. Brux.,} L.M.S.S.A. LOND., D.T.M. LIVERP.,

MEDICAL OFFICER, SWINTON AND STALYBRIDGE ANTE-NATAT CLINICS ; LATE SUPT. GOVT. AREAS G.M. HOSPITAL, TRANSVAAL, S.A.; AND

Beryard Hirson, L.R.C.S. \& P. Irec., L.M.

AlthougH Ascaris lumbricoides is a common parasite of man, and found in most parts of the world, the following case is interesting owing to the severity of the symptoms and the patient's rapid recovery after removal of the cause:-

Mrs. M., a Turkish woman, aged 25, a native of Aleppo, only three months resident in England, and three months pregnant (primipara), was seen by us complaining of constant nausea, cough, loss of flesh, and weakness. As nothing definite was found, with the exception of a few moist râles in both lungs, accounted for by a recent bad cold, her condition was put down to her being enceinte.

A few days afterwards we again saw her, in answer to an urgent call. She had been coughing and vomiting constantly for about 24 hours, and was so weak that she was unable to leave her bed.

Examination.-Patient was very pale, face drawn and anxious, supra- and infra-clavicular regions and intercostal spaces were hollow, pelvic bones prominent. Abdomen flat, limbs correspondingly thin-in fact, patient presented a typical picture of starvation. She hiccoughed, coughed, and vomited during examination. The temperature was subnormal; vomited matter was mucous, bile-stained, and contained clots of fresh blood, as did the fæecs, diarrhoea having set in. Mouth clean, tongue furred but moist, chest normal except for the few moist râles previously noted heart sounds were normal, but weak and rapid ; pulse weak on palpation of abdomen general tenderness was found, especially marked over region of stomach and umbilicus. No rigidity, no swelling of joints or enlargement of glands.

Course of Case. A provisional diagnosis of acute gastroenteritis was made until urine and fæces could be examined and a bismuth carb., salol, and morph. mixture prescribed ; peptonised food with brandy was given in very small quan tities at frequent intervals, as it was imperative that the patient should obtain some nourishment at once. Later in the day, as all food was returned, nothing but ice to suck and small frequent doses of brandy were given. In the evening, during an attack of coughing and vomiting, an Ascaris lumbricoides was expelled; this eased the coughing and straining considerably, and an houx or two afterwards another worm was passed in the fæces. We then decided to treat the case as one of acute ascariasis. On the second day a hypodermic injection of strych. c. digitalin was given, and a draught of santonin $g r$. $\forall$, salol. gr. $\nabla$. in syrup of senna administered. This was followed later by calomel gr.v. Vomiting and cough were less, and more nourishment was retained. During the morning of the third day a free evacuation of the bowels resulted in the expulsion of eight large worms, two more being vomited during the night. On the fourth day a second santonin and salol draught was given. followed by calomel gr. iv. free evacuation resulted in the expulsion of six more worms, and the patient only had two attacks of coughing and vomiting, each time expelling two ascarides. The evening of the fifth day brought to light three more worms during attacks of coughing and vomiting, and three others were passed in the fæces. On examining the fæces passed, in addition to the ascarides, two whip worms (Trichuris trichiura) were found. The patient, although very 